\title{
Review Article \\ Basic Generic Properties of Regular Rotating Black Holes and Solitons
}

\author{
Irina Dymnikova ${ }^{1,2}$ and Evgeny Galaktionov ${ }^{1}$ \\ ${ }^{1}$ A. F. Ioffe Physico-Technical Institute, Politekhnicheskaja 26, St. Petersburg 194021, Russia \\ ${ }^{2}$ Department of Mathematics and Computer Science, University of Warmia and Mazury, Sloneczna 54, 10-710 Olsztyn, Poland
}

Correspondence should be addressed to Irina Dymnikova; irina@uwm.edu.pl

Received 30 April 2017; Revised 14 June 2017; Accepted 29 August 2017; Published 16 October 2017

Academic Editor: Stephen C. Anco

Copyright (C) 2017 Irina Dymnikova and Evgeny Galaktionov. This is an open access article distributed under the Creative Commons Attribution License, which permits unrestricted use, distribution, and reproduction in any medium, provided the original work is properly cited.

\begin{abstract}
We present a systematic description of the basic generic properties of regular rotating black holes and solitons (compact nonsingular nondissipative objects without horizons related by self-interaction and replacing naked singularities). Rotating objects are described by axially symmetric solutions typically obtained by the Gürses-Gürsey algorithm, which is based on the Trautman-Newman techniques and includes the Newman-Janis complex transformation, from spherically symmetric solutions of the Kerr-Schild class specified by $T_{t}^{t}=T_{r}^{r}\left(p_{r}=-\rho\right)$. Regular spherical solutions of this class satisfying the weak energy condition have obligatory de Sitter center. Rotation transforms de Sitter center into the equatorial de Sitter vacuum disk. Regular solutions have the Kerr or KerrNewman asymptotics for a distant observer, at most two horizons and two ergospheres, and two different kinds of interiors. For regular rotating solutions originated from spherical solutions satisfying the dominant energy condition, there can exist the interior $\mathcal{S}$-surface of de Sitter vacuum which contains the de Sitter disk as a bridge. In the case when a related spherical solution violates the dominant energy condition, vacuum interior of a rotating object reduces to the de Sitter disk only.
\end{abstract}

\section{Introduction}

Presented in the current literature, regular rotating solutions [1-10] are obtained from regular spherical solutions with using the Newman-Janis complex translation [11]. In [12], it was shown that the Newman-Janis translation works for algebraically special metrics which belong to the Kerr-Schild class [13] and can be presented as $g_{\mu \nu}=\eta_{\mu \nu}+2 f(r) k_{\mu} k_{v}$, where $\eta_{\mu \nu}$ is the Minkowski metric and $k_{\mu}$ are principal null congruences. More general approach, with using basic properties of metrics from the Kerr-Schild class, was applied for obtaining the axially symmetric solutions in the noncommutative geometry [14]. [13]

Spherical metrics of the Kerr-Schild class have the form

$$
d s^{2}=g(r) d t^{2}-\frac{d r^{2}}{g(r)}-r^{2}\left(d \theta^{2}+\sin ^{2} \theta d \phi^{2}\right),
$$

where

$$
g(r)=1-\frac{2 \mathscr{M}(r)}{r} ; \quad \mathscr{M}(r)=4 \pi \int_{0}^{r} \rho(x) x^{2} d x,
$$

and are generated by stress-energy tensors satisfying [15-18]

$$
T_{t}^{t}=T_{r}^{r} .
$$

The energy density is $\rho=T_{t}^{t}$, the radial pressure $p_{r}=-T_{r}^{r}$, and the transversal pressure $p_{\perp}=-T_{\theta}^{\theta}=-T_{\phi}^{\phi}$. Equation (3) represents the radial part of the $r$-dependent equation of state, $p_{r}=-\rho$. The contracted Bianchi identities $\left(T_{\mu ; \nu}^{\nu}=0\right)$ yield the second part of the $r$-dependent equation of state, $p_{\perp}=$ $-\rho-(r / 2) \rho^{\gamma}[15,19]$.

All regular spherical solutions of this class, satisfying the weak energy condition (nonnegative density as measured by any local observer on a time-like curve), have obligatory de Sitter center, $T_{\mu}^{\nu} \rightarrow \rho_{0} \delta_{\mu}^{\nu} ; g(r) \rightarrow 1-r^{2} / r_{0}^{2}$ as $r \rightarrow 0$ with $r_{0}^{2}=3 /\left(8 \pi G \rho_{0}\right)[18,20]$.

Gürses and Gürsey have found that the algebraically special metrics of the Kerr-Schild class can be presented in the Lorentz covariant coordinate system, developed the general approach based on the complex Trautman-Newman techniques (which include the Newman-Janis translation), and 
derived the axially symmetric metric in the general, modelindependent form. In the Boyer-Lindquist coordinates the metric reads [12]

$$
\begin{aligned}
d s^{2}= & \frac{2 f-\Sigma}{\Sigma} d t^{2}+\frac{\Sigma}{\Delta} d r^{2}+\Sigma d \theta^{2}-\frac{4 a f \sin ^{2} \theta}{\Sigma} d t d \phi \\
& +\left(r^{2}+a^{2}+\frac{2 f a^{2} \sin ^{2} \theta}{\Sigma}\right) \sin ^{2} \theta d \phi^{2}, \\
\Delta= & r^{2}-2 f(r)+a^{2} \\
\Sigma= & r^{2}+a^{2} \cos ^{2} \theta .
\end{aligned}
$$

It involves a master function

$$
f(r)=r \mathscr{M}(r)
$$

which comes from a spherically symmetric solution (2). For $f(r)=M r-q^{2} / 2$, it describes the Kerr-Newman geometry [21], where $M$ is the mass parameter and $q$ is the electric charge. The case $q=0$ corresponds to the Kerr geometry [22]. Each of these two geometries can have at most two horizons, the solutions without horizons describe naked singularities.

The parameter $r$ in (4) is defined as an affine parameter along a principal null congruence. The surfaces of constant $r$ are the confocal ellipsoids of revolution [23]

$$
r^{4}-\left(x^{2}+y^{2}+z^{2}-a^{2}\right) r^{2}-a^{2} z^{2}=0
$$

which degenerate, for $r=0$, to the equatorial disk

$$
\begin{array}{r}
x^{2}+y^{2} \leq a^{2}, \\
z=0,
\end{array}
$$

centered on the symmetry axis and bounded by the ring $x^{2}+$ $y^{2}=a^{2}, z=0$ (in the Kerr and Kerr-Newman geometries, it comprises their ring singularities).

Regular axially symmetric solutions satisfy condition (3) in the corotating reference frame $[24,25]$ and describe regular rotating objects, asymptotically Kerr or Kerr-Newman for a distant observer with the mass parameter $M=\mathscr{M}(r \rightarrow \infty)$. Solutions of this class describe regular rotating black holes and spinning solitons with de Sitter vacuum interiors [2528]. Rotation transforms de Sitter center of regular spherical solutions into de Sitter vacuum disk (8). All regular solutions presented in the literature belong to this class. Most of them are devoted to study of particular solutions specified by a choice of the mass function $\mathscr{M}(r)$ in (2).

In this paper, we present a systematic review of the basic generic properties of regular rotating black holes and spinning solitons replacing naked singularities and defined in the spirit of Coleman lumps [29] as nonsingular nondissipative objects without horizons keeping themselves together by their own self-interaction. For convenience and transparency in analysis of various aspects of generic behavior, we use the geometrized units $c=G=1$. Conversion factors and instructions for transition to different nongeometrized units can be found in [30].

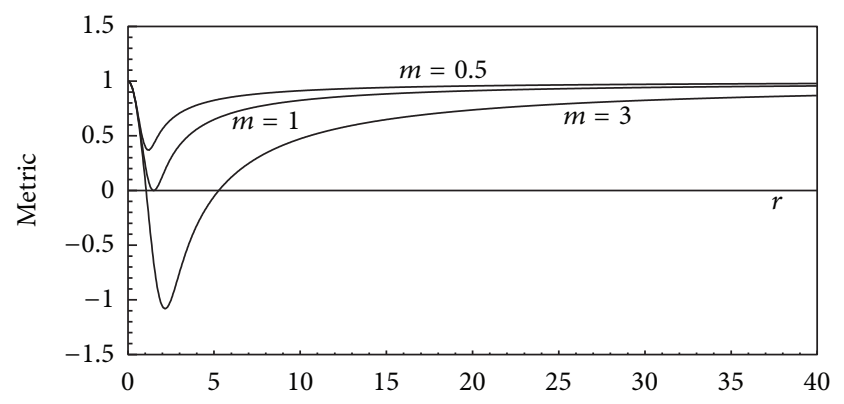

FIGURE 1: Typical behavior of spherical metric function $g(r)$. Here $m$ is the mass parameter $M$ normalized to its certain critical value which specifies the double-horizon state.

The paper is organized as follows. In Section 2, we present horizons, ergospheres, and ergoregions. Section 3 is devoted to basic physical properties of regular interiors. Section 4 outlines behavior of electromagnetic fields in the case of electrically charged regular black holes and electromagnetic solitons. In Section 5, we summarize and discuss the results.

\section{Horizons, Ergospheres, and Ergoregions}

The basic features of regular rotating objects are closely related to generic properties of related regular spherical solutions (1)-(2).

Horizons are defined by $\Delta(r)=0$. The function $\Delta(r)$ can be written as

$$
\Delta(r)=r^{2}+a^{2}-2 f(r)=a^{2}+r^{2} g(r) .
$$

It follows that $\Delta=a^{2}$ at zero points of the metric function $g(r), g(r)=0$, and evolves from $\Delta=a^{2}$ as $r=0$ to $\Delta \rightarrow \infty$ as $r \rightarrow \infty$.

A spherical metric function $g(r)$ in (2) in the asymptotically flat case can have at most two zero points and one minimum between them [18]. Typical behavior of a spherical metric function $g(r)$ is shown in Figure 1.

Derivatives of $\Delta$ are $\Delta^{\prime}=2 r g(r)+r^{2} g^{\prime}(r) ; \Delta^{\prime \prime}=2 g(r)+$ $4 r g^{\prime}(r)+r^{2} g^{\prime \prime}(r)$. At $r=0$ derivatives take the values $\Delta^{\prime}=0$; $\Delta^{\prime \prime}=2$ and the function $\Delta$ has the minimum, $\Delta=a^{2}$. Next it grows and can have maximum at a certain value $r_{m}<r_{1}$, where $r_{1}$ is the 1st zero of the function $g(r)$. At the maximum $\Delta^{\prime}\left(r_{m}\right)=0$ and hence $g^{\prime}\left(r_{m}\right)=-2 g\left(r_{m}\right) / r_{m}$, second derivative $\Delta^{\prime \prime}\left(r_{m}\right)=-6 g\left(r_{m}\right)+r_{m}^{2} g^{\prime \prime}\left(r_{m}\right)<0$. After passing the maximum, decreasing $\Delta$ achieves $\Delta=a^{2}$ at the 1st zero of $g(r)$; then it will achieve this value at the 2 nd zero point $r_{2}$ of $g(r)$. The function $g(r)<0$ between its zeros and has the minimum. In this region, $g^{\prime}(r)$ is first negative and then passes zero and becomes positive; hence, $g^{\prime \prime} \geq 0$ everywhere between zeros of $g(r)$, while $g \leq 0$; as a result, $\Delta^{\prime \prime} \geq 0$ everywhere in this region, so that the function $\Delta(r)$ can have only minimum and only one. At $r>r_{2}$ we have $g(r)>0$ and $g^{\prime}>0$ so that $\Delta$ cannot vanish. Since the function $g(r)$ has at most two zero points, axially symmetric space-time can have at most two horizons, the event horizon $r_{+}$and the internal Cauchy horizon $r_{-}<r_{+}[27,28]$. 


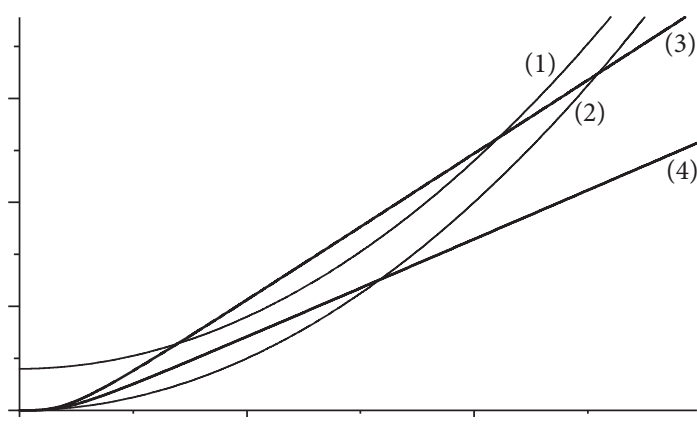

(a)

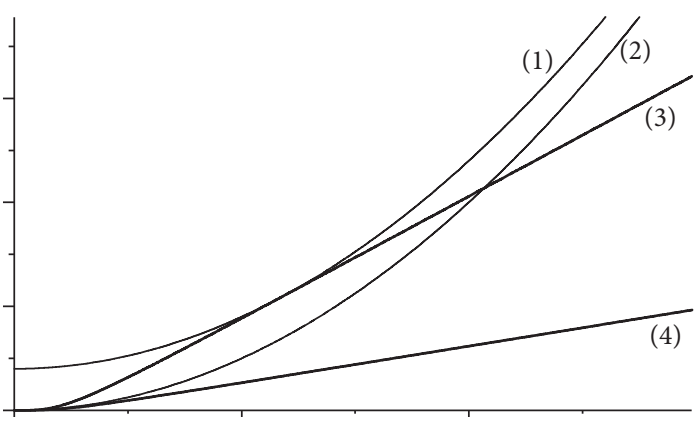

(b)

FIGURE 2: Four cases for the existence of ergospheres and ergoregions.

Ergosphere is a surface of a static limit $g_{t t}=0$ given by

$$
g_{t t}(r, \theta)=r^{2}+a^{2} \cos ^{2} \theta-2 f(r)=0 .
$$

Ergoregions are defined by $g_{t t}<0$ which makes possible extraction of rotational energy (see, e.g., [4] and references therein).

Each point of the ergosphere belongs to some of confocal ellipsoids (7) covering the whole space as the coordinate surfaces $r=$ const. The Cartesian coordinates satisfy $z=$ $r \cos \theta ; x^{2}+y^{2}=\left(a^{2}+r^{2}\right) \sin ^{2} \theta$. It follows from (10) that $z^{2}=\left(2 r^{2} f(r)-r^{4}\right) / a^{2}$ on the ergosphere.

There are four cases for the existence of ergospheres and ergoregions shown in Figure 2. The width of the ergosphere at a certain $z$ is $x^{2}+y^{2}=\left(a^{2}+r^{2}\right)\left(1-z^{2} / r^{2}\right)$. In the equatorial plane $x^{2}+y^{2}=a^{2}+r^{2}$ and $z^{2}=0 \rightarrow 2 f(r)-r^{2}=0$. For any regular density profile, the function $f(r)$ (curves (3), (4) in Figure 2) is everywhere nonnegative and monotonically grows from $f(r)=4 \pi \rho_{0} r^{4} / 3$ at $r \rightarrow 0$ [18]. Ergosphere exists when the curve $u=2 f(r)$ intersects or touches the parabola $u=r^{2}$ (curves (2) in Figure 2). It is evident that in this case the curve $u=2 f(r)$ intersects also the (situated above) parabola $u=r^{2}+a^{2} \cos ^{2} \theta$ for a given $\theta$ (curves (1)).

In the black hole case, ergospheres and ergoregions exist for any density profile (the curve (3a) for the case of two horizons and the curve (3b) for the double-horizon case). In the case of a soliton the existence of ergospheres depends on the density profile. Solitons can have two ergospheres and ergoregion between them (the curve (4a)), one ergosphere and ergoregion involving the whole interior (the curve (4b)), or no ergospheres $[27,28]$.

\section{Internal Structure of Regular Rotating Objects}

3.1. Density and Pressures. The anisotropic stress-energy tensor responsible for (4) can be written in the form [12]

$$
T_{\mu \nu}=\left(\rho+p_{\perp}\right)\left(u_{\mu} u_{\nu}-l_{\mu} l_{\nu}\right)+p_{\perp} g_{\mu \nu}
$$

in the orthonormal tetrad

$$
\begin{aligned}
u^{\mu} & =\frac{1}{\sqrt{ \pm \Delta \Sigma}}\left[\left(r^{2}+a^{2}\right) \delta_{0}^{\mu}+a \delta_{3}^{\mu}\right], \\
l^{\mu} & =\sqrt{\frac{ \pm \Delta}{\Sigma}} \delta_{1}^{\mu}, \\
n^{\mu} & =\frac{1}{\sqrt{\Sigma}} \delta_{2}^{\mu}, \\
m^{\mu} & =\frac{-1}{\sqrt{\Sigma} \sin \theta}\left[a \sin ^{2} \theta \delta_{0}^{\mu}+\delta_{3}^{\mu}\right] .
\end{aligned}
$$

The sign plus refers to the regions outside the event horizon and inside the Cauchy horizon where the vector $u^{\mu}$ is timelike, and the sign minus refers to the regions between the horizons where the vector $l^{\mu}$ is time-like. The vectors $m^{\mu}$ and $n^{\mu}$ are space-like in all regions.

The eigenvalues of the stress-energy tensor (11), calculated in the corotating references frame where each of ellipsoidal layers of constant $r$ rotates with the angular velocity $\omega(r)=$ $u^{\phi} / u^{t}=a /\left(r^{2}+a^{2}\right)[24]$, are defined by

$$
\begin{aligned}
& T_{\mu \nu} u^{\mu} u^{\nu}=\rho(r, \theta) \\
& T_{\mu \nu} l^{\mu} l^{\nu}=p_{r}=-\rho \\
& T_{\mu \nu} n^{\mu} n^{\nu}=T_{\mu \nu} m^{\mu} m^{\nu}=p_{\perp}(r, \theta)
\end{aligned}
$$

in the regions outside the event horizon and inside the Cauchy horizon where density is defined as the eigenvalue corresponding to the time-like eigenvector $u^{\mu}$.

The eigenvalues of the stress-energy tensor are related to the master function $f(r)$ as [24]

$$
\begin{aligned}
\kappa \Sigma^{2} \rho(r, \theta) & =2\left(f^{\prime} r-f\right) ; \\
\kappa \Sigma^{2} p_{\perp}(r, \theta) & =2\left(f^{\prime} r-f\right)-f^{\prime \prime} \Sigma,
\end{aligned}
$$


where $\kappa=8 \pi G$. It follows that

$$
\begin{aligned}
\rho(r, \theta) & =\frac{r^{4}}{\Sigma^{2}} \widetilde{\rho}(r) ; \\
p_{r} & =-\rho ; \\
p_{\perp}(r, \theta) & =\left(\frac{r^{4}}{\Sigma^{2}}-\frac{2 r^{2}}{\Sigma}\right) \tilde{\rho}(r)-\frac{r^{3}}{2 \Sigma} \tilde{\rho}^{\prime}(r),
\end{aligned}
$$

where $\tilde{\rho}(r)$ is the related spherical density. In the corotating frame, we thus have

$$
\left(p_{\perp}+\rho\right)=2\left(\frac{r^{4}}{\Sigma^{2}}-\frac{r^{2}}{\Sigma}\right) \tilde{\rho}(r)-\frac{r^{3}}{2 \Sigma} \widetilde{\rho}^{\prime}(r) .
$$

3.2. De Sitter Disk. Near the disk (8) the function $f(r)$ in (4) approaches de Sitter asymptotic $2 f(r) \rightarrow r^{4} / r_{0}^{2} ; r_{0}^{2}=$ $3 /\left(8 \pi G \widetilde{\rho}_{0}\right)$. Taking into account $\Sigma=\left(r^{4}+a^{2} z^{2}\right) r^{-2}$, we get in the equatorial plane $r^{2} / \Sigma \rightarrow 1$ as $z \rightarrow 0$, and (16) reduces to $[25]$

$$
\left(p_{\perp}+\rho\right)=-\frac{r \tilde{\rho}^{\prime}(r)}{2} .
$$

For the regular spherical solutions satisfying the weak energy condition $\widetilde{\rho}^{\prime} \leq 0$, and regularity requires $r \widetilde{\rho}^{\prime}(r) \rightarrow 0$ as $r \rightarrow 0$ [18]. Then $p_{\perp}=-\rho$, and the equation of state on the disk

$$
p=-\rho
$$

represents the rotating de Sitter vacuum in the corotating frame [25]. According to (15), the density in the equatorial plane is $\rho(r, \theta)=\widetilde{\rho}(r)$. When $r \rightarrow 0, \widetilde{\rho}(r) \rightarrow \widetilde{\rho}_{0}$, so that on the equatorial disk $\rho(r, \theta)=\widetilde{\rho}_{0}(r)$.

3.3. Two Types of Interiors. In [24], it was suggested that rotation should inevitably lead to violation of the weak energy condition in interior regions of regular rotating structures. Violation of the weak energy condition was found for regular rotating solutions obtained with the Newman-Janis algorithm from particular spherical metrics $[3,4]$ and for a solution found by postulating a metric $g_{\mu \nu}$ and calculating $T_{\mu \nu}$ from the Einstein equations [5].

In our papers $[27,28]$ we have studied the weak energy condition for rotating regular structures on general setting and found the existence of two kinds of regular interiors, one preserving and the other violating the weak energy condition.

The weak energy condition is valid if and only if $\rho \geq 0$ and $p_{\perp}+\rho \geq 0$ [31]. The first condition is satisfied according to (15). The general equation (16) for $p_{\perp}+\rho$ can be written as

$$
p_{\perp}+\rho=\frac{2 r^{2}}{\Sigma^{2}}\left(\frac{\Sigma r}{4}\left|\tilde{\rho}^{\prime}\right|-\tilde{\rho} a^{2} \cos ^{2} \theta\right) .
$$

It implies a possibility for generic violation of the weak energy condition beyond the de Sitter vacuum surface $\mathcal{S}(r, z)=0$, on which $p_{\perp}+\rho=0$ and the right-hand side in (19) can change

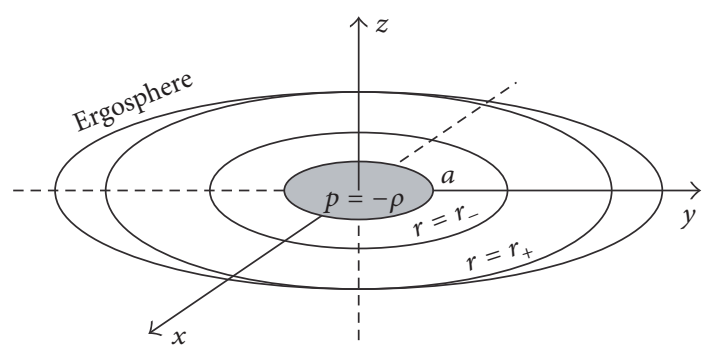

FIGURE 3: First type of the de Sitter interior for regular rotating objects.

its sign. It can be expressed through the pressure of a related spherical solution, $\widetilde{p_{\perp}}=-\tilde{\rho}-r \tilde{\rho}^{\prime} / 2$, which gives

$$
\begin{aligned}
p_{\perp}+\rho & =\frac{r\left|\tilde{\rho}^{\prime}\right|}{2 \Sigma^{2}} \mathcal{S}(r, z) ; \\
\mathcal{S}(r, z) & =r^{4}-z^{2} P(r) ; \\
P(r) & =\frac{2 a^{2}}{r\left|\widetilde{\rho}^{\prime}\right|}\left(\widetilde{\rho}-\widetilde{p_{\perp}}\right) .
\end{aligned}
$$

As we see, the existence of vacuum surfaces and hence the violation of the weak energy condition beyond them is possible if and only if a related spherical solution satisfies the dominant energy condition [31] $\left(\widetilde{\rho} \geq \widetilde{p_{k}}\right.$ in $\left.(20)\right)$. In the case when a related spherical solution violates the dominant energy condition, the weak energy condition is preserved for a rotating solution.

Regular Interiors Satisfying the Weak Energy Condition. In this case $\widetilde{\rho}<\widetilde{p}_{\perp}$, hence $P(r) \leq 0$, and the function $\mathcal{S}(r, z)$ in (20) vanishes only at approaching the disk $r=0$. In this case the interior region looks as shown in Figure 3 [28] where we also plotted two horizons defined by the roots of the equation $r^{2}+a^{2}-2 f(r)=0$, the event and the internal horizons $r=$ $r_{+}, r=r_{-}$, respectively, and the ergosphere satisfying $r^{2}+$ $a^{2} \cos ^{2} \theta-2 f(r)=0$.

Regular Interiors Violating the Weak Energy Condition. In the case when $P(r) \geq 0$, there can in principle exist $\mathcal{S}$-surface at which $p_{\perp}+\rho=0$ and beyond which the weak energy condition is violated. On the $\mathcal{S}$-surface $z^{2}=r^{4} / P(r)$ and hence $r^{2}=|z| \sqrt{P(r)}$. The squared width of the $\mathcal{S}$-surface, $W_{\mathcal{S}}^{2}=\left(x^{2}+y^{2}\right)_{\mathcal{S}}=\left(a^{2}+|z| \sqrt{P(r)}\right)(1-|z| / \sqrt{P(r)})$. The squared width of the ellipsoid $r=|z|_{\max }=r_{e}$ is $W_{e}^{2}=$ $\left(a^{2}+r_{e}^{2}\right)\left(1-z^{2} / r_{e}^{2}\right)$. The difference $W_{e}^{2}-W_{\mathcal{S}}^{2}=\left(a^{2}|z| / \sqrt{P(r)}+\right.$ $\left.r_{e}^{2}\right)\left(1-|z| \sqrt{P(r)} / r_{e}^{2}\right)$. Assume that $W_{\mathcal{S}}>W_{e}$ for some value of $z$; it means that there exists a ring specified by $|z|<|z|_{\text {max }}$ which belongs to the ellipsoid $r=r_{e}$, where these two surfaces intersect and where thus $|z| \sqrt{P\left(r_{e}\right)} / r_{e}^{2}=1$. As a result, $|z|=$ $r_{e}^{2} / \sqrt{P\left(r_{e}\right)}=|z|_{\max }$, so that these two surfaces can only touch each other on the poles and hence never cross. It follows that the $\mathcal{S}$-surface is always entirely confined within the ellipsoid 


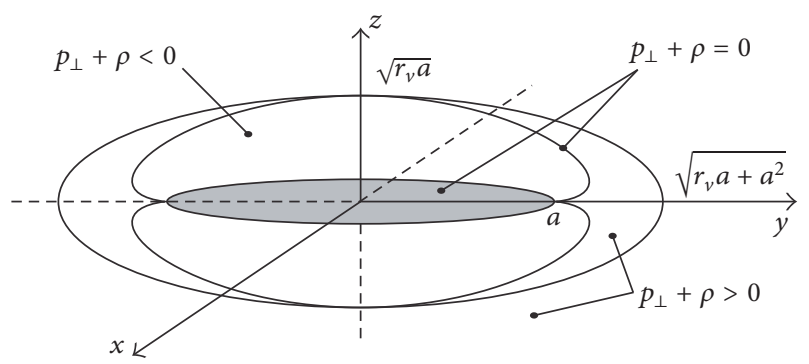

FIGURE 4: Second type of interior for regular rotating black holes and solitons.

defined by $|z|_{\max }=r_{e}=r_{e}^{2} / \sqrt{P\left(r_{e}\right)}$ and contains the disk $r=0$ as a bridge.

For regular spherical solutions $r \tilde{\rho}^{\prime} \rightarrow 0, \tilde{p}_{\perp} \rightarrow-\tilde{\rho}$ as $r \rightarrow 0$, and in the most general case $P(r) \rightarrow A^{2} r^{-(n+1)}$ with the integer $n \geq 0$ as $r \rightarrow 0$. As a result the derivative of $W_{\delta}(z)$ near $z \rightarrow 0$ behaves as $z^{-(n+3) /(n+5)}$ and goes to $\pm \infty$ as $z \rightarrow 0$, so that the function $W_{\mathcal{\delta}}(z)$ has the cusp at approaching the disk and at least two symmetric (with respect to the equatorial plane) maxima between $z= \pm\left|z_{\max }\right|$ and $z=0$. Typical form of the de Sitter $\mathcal{S}$-surface for the second type of interior is shown in Figure 4 [27, 28].

In the cavities between its upper and down boundaries and the bridge $p_{\perp}+\rho<0$, the weak energy condition is violated and cavities are filled with a phantom energy which is defined by the condition $p+\rho<0$ [32]; in the considered case, it is satisfied for $p_{\perp}$.

As we have shown, the form of the de Sitter $\mathcal{S}$-surfaces is generic. Here we plotted it for the particular exact solution $[27,28]$ which originates from the spherically symmetric solution [33]

$$
\begin{aligned}
\tilde{\rho} & =\frac{B^{2}}{\left(r^{2}+r_{v}^{2}\right)^{2}} ; \\
r_{v} & =\frac{\pi}{8} \frac{B^{2}}{M} ; \\
f(r) & =\frac{r_{g}}{\pi}\left(\arctan \frac{r}{r_{v}}-\frac{r r_{v}}{r^{2}+r_{v}^{2}}\right),
\end{aligned}
$$

with a phenomenologically regularized Newtonian [28] or Coulomb [27] density profile, dependent on identification of the parameter $B^{2} / r_{v}^{4}=\widetilde{\rho}_{0}$ which represents the cut-off on selfinteraction energy by the de Sitter vacuum density $\widetilde{\rho}_{0}$ at $r=0$. The density $\widetilde{\rho}_{0}$ is related to the mass $M$ by the formula $M=$ $(4 \pi / 3) \widetilde{\rho}_{0} r_{0}^{2} r_{g}=(4 \pi / 3) \widetilde{\rho}_{0} r_{*}^{3}$. The length scale $r_{*}=\left(r_{0}^{2} r_{g}\right)^{1 / 3}$ which appears here in the natural way is characteristic for a geometry with the de Sitter interior and the Schwarzschild exterior $[15,17,19]$.

Relation between the width of the $\mathcal{S}$-surface in the equatorial plane $W_{\mathcal{S}}=a$ and its height $H_{\mathcal{S}}=|z|_{\max }=\sqrt{a r_{v}}$ defines the form of the $\mathcal{S}$-surface by the oblateness parameter $\eta=H_{\mathcal{S}} / W_{\mathcal{S}}$. For $\eta<1$ the $\mathcal{S}$-surface is oblate (shown in Figure 4). It can be the case of electrically neutral quickly rotating de Sitter-Kerr black holes and solitons [28]. For $\eta>1$ the de Sitter $\mathcal{S}$-surface is prolate; it may be the case of a slowly rotating black hole (more details in $[27,28]$ ).

\section{Electrically Charged Regular Black Holes and Solitons}

4.1. Basic Equations. Objects related by the electromagnetic and gravitational interaction are governed by nonlinear electrodynamics coupled to gravity.

Nonlinear electrodynamics was proposed by Born and Infeld as founded on two basic principles: to consider electromagnetic field and particles within the frame of one physical entity which is electromagnetic field and to avoid letting physical quantities become infinite [34]. In this theory, finite values of the field energy are achieved by imposing an upper limit on the electric field, but geometry remains singular.

The program based on two above principles can be realized in nonlinear electrodynamics coupled to gravity (NED-GR) in the frame of the standard minimal coupling of gravitational and electromagnetic field, that is, without introducing some hypothetical nonminimal coupling which could be essential at the Planck energies, since in the modern string/M-theories nonlinear electrodynamics appears as lowenergy effective limits $[35,36]$.

Electrically charged regular objects are described by the source-free NED-GR equations. The source of the gravitational fields in the Einstein equations is the stress-energy tensor of a nonlinear electromagnetic field.

For any gauge-invariant Lagrangian $\mathscr{L}(F)$, stress-energy tensor of electromagnetic field reads

$$
\kappa T_{\nu}^{\mu}=-2 \mathscr{L}_{F} F_{\nu \alpha} F^{\mu \alpha}+\frac{1}{2} \delta_{v}^{\mu} \mathscr{L},
$$

where $F_{\mu \nu}=\partial_{\mu} A_{\nu}-\partial_{\nu} A_{\mu}$ and $\mathscr{L}_{F}=d \mathscr{L} / d F$. In the spherically symmetric case, it has the algebraic structure (3) and metrics belong to the Kerr-Schild class (2).

Nonlinear electrodynamics minimally coupled to gravity is described by the action

$$
\begin{aligned}
& S=\frac{1}{16 \pi G} \int d^{4} x \sqrt{-g}[R-\mathscr{L}(F)] \\
& F=F_{\mu \nu} F^{\mu \nu}
\end{aligned}
$$

where $R$ is the scalar curvature and $g$ is the determinant of the metric tensor. The Lagrangian $\mathscr{L}(F)$ is an arbitrary function of $F$ which should have the Maxwell limit, $\mathscr{L} \rightarrow F, \mathscr{L}_{F} \rightarrow 1$, in the weak field regime. Variation with respect to $A^{\mu}$ and $g_{\mu \nu}$ yields the dynamic equations

$$
\begin{aligned}
\nabla_{\mu}\left(\mathscr{L}_{F} F^{\mu \nu}\right) & =0 ; \\
\nabla_{\mu}{ }^{*} F^{\mu \nu} & =0,
\end{aligned}
$$

where ${ }^{*} F^{\mu \nu}=(1 / 2) \eta^{\mu \nu \alpha \beta} F_{\alpha \beta} ; \eta^{0123}=-1 / \sqrt{-g}$, and the Einstein equations $G_{\mu \nu}=-\kappa T_{\mu \nu}$ with the electromagnetic source given by the stress-energy of a nonlinear electromagnetic field (22). 
4.2. Generic Features of the Lagrange Dynamics. The key point is that regularity requires $F \rightarrow-0$ as $r \rightarrow 0$, while the Maxwell limit implies $F \rightarrow-0$ at infinity. Indeed, in the spherically symmetric case $F=-2 q^{2} / \mathscr{L}_{F}^{2} r^{4}$ [37], which results in $F \mathscr{L}_{F}^{2} \rightarrow-\infty$ as $r \rightarrow 0$. The structure of stressenergy tensor implies $p_{\perp}+\rho=F \mathscr{L}_{F}$, regular solutions have obligatory de Sitter center, where $p_{\perp}+\rho=0$, and hence $F \mathscr{L}_{F}=0$ [33]. Requirement of regularity demands that $\mathscr{L}_{F} \rightarrow \infty$ and $F \rightarrow-0$ when $r \rightarrow 0$. Nonmonotonic behavior of the invariant $F$ leads inevitably to branching of a Lagrangian $\mathscr{L}(F)[33,37]$.

This problem is solved by the correct description of the Lagrange dynamics for regular electrically charged structures by the nonuniform variational problem with proper internal boundary conditions on the surface where the invariant $F$ has the minimum [38]. The nonuniform variational problem is described by the action

$$
\begin{aligned}
S= & S_{\text {int }}+S_{\text {ext }}=\frac{1}{16 \pi}\left[\int_{\Omega_{\text {int }}}\left(R-\mathscr{L}_{\text {int }}(F)\right) \sqrt{-g} d^{4} x\right. \\
& \left.+\int_{\Omega_{\text {ext }}}\left(R-\mathscr{L}_{\text {ext }}(F)\right) \sqrt{-g} d^{4} x\right] .
\end{aligned}
$$

Each part of the manifold, $\Omega_{\text {int }}$ and $\Omega_{\text {ext }}$, is confined by the space-like hypersurfaces $t=t_{\text {in }}$ and $t=t_{\text {fin }}$ and by the timelike 3-surface at infinity, where electromagnetic fields vanish in the Maxwell limit. Internal boundary between $\Omega_{\text {int }}$ and $\Omega_{\text {ext }}$ is defined as a time-like hypersurface $\Sigma_{c}$ at which the field invariant $F$ achieves its extremum.

In the case of the minimal coupling variation in the action (25) results in the dynamical equations (24) in both $\Omega_{\text {int }}$ and $\Omega_{\text {ext }}$, and in the boundary conditions on the surface $\Sigma_{c}[38]$

$$
\begin{aligned}
& \int_{\Sigma_{c}}\left(\mathscr{L}_{F(\text { int })} F_{\mu \nu(\text { int })}-\mathscr{L}_{F(\text { ext) }} F_{\mu \nu(\text { ext })}\right) \sqrt{-g} \delta A^{\mu} d \sigma^{\nu}=0 \\
& \mathscr{L}_{\text {(int) }}-2 \mathscr{L}_{F(\text { int })} F_{\text {int }}=\mathscr{L}_{(\text {ext })}-2 \mathscr{L}_{F(\text { ext })} F_{\text {ext }} .
\end{aligned}
$$

The particular solution (21) can be obtained with the NEDGR Lagrangians.

$\mathscr{L}_{\text {int }}(F)=2\left(1-z_{2}(\beta)\right) /\left(z_{2}(\beta)+1\right)^{3}$ with $z_{2}=-(2 \sqrt{1-6 \beta} /$ $3 \beta) \cos (\alpha / 3+\pi / 3)-1+1 / 3 \beta, \mathscr{L}_{\text {ext }}(F)=2\left(1-z_{1}(\beta)\right) /\left(z_{1}(\beta)+\right.$ $1)^{3}$ with $z_{1}=(2 \sqrt{1-6 \beta} / 3 \beta) \cos (\alpha / 3)-1+1 / 3 \beta$, where the invariant $F$ is normalized on $q^{2} / r_{q}^{4}, r_{q}=(\pi / 8)\left(q^{2} / m\right) ; \beta=$ $\sqrt{-F / 2}, \beta \in(0,4 / 27)$, and $\alpha=\arccos \left(\left(2-18 \beta+27 \beta^{2}\right) / 2(1-\right.$ $\left.6 \beta)^{3 / 2}\right)[38]$.

The same behavior of the invariant $F$ is generic for regular axisymmetric solutions, $F=-0$ on the disk $r=0$ [27] and in the Maxwell limit at $r \rightarrow \infty$. As a result, the basic generic feature of the regular electrically charged structures is the existence of a characteristic surface separating regions described by different Lagrangians of the nonuniform variational problem.

4.3. Dynamics of Electromagnetic Fields. Nonzero field components compatible with the axial symmetry are $F_{01}$,
$F_{02}, F_{13}, F_{23}$. In geometry with the metric (4) they are related by

$$
\begin{aligned}
& F_{31}=a \sin ^{2} \theta F_{10} ; \\
& F_{23}=\left(r^{2}+a^{2}\right) F_{02} .
\end{aligned}
$$

Due to (27), (24) form the system of four equations for two independent functions [27]

$$
\begin{aligned}
& \frac{\partial}{\partial r}\left[\left(r^{2}+a^{2}\right) \sin \theta \mathscr{L}_{F} F_{10}\right]+\frac{\partial}{\partial \theta}\left[\sin \theta \mathscr{L}_{F} F_{20}\right]=0, \\
& \frac{\partial}{\partial r}\left[\frac{1}{\sin \theta} \mathscr{L}_{F} F_{31}\right]+\frac{\partial}{\partial \theta}\left[\frac{1}{\left(r^{2}+a^{2}\right) \sin \theta} \mathscr{L}_{F} F_{32}\right] \\
& \quad=0 \\
& \frac{\partial F_{23}}{\partial r}+\frac{\partial F_{31}}{\partial \theta}=0, \\
& \frac{\partial F_{01}}{\partial \theta}+\frac{\partial F_{20}}{\partial r}=0 .
\end{aligned}
$$

Solutions to this system should satisfy the compatibility condition [27]

$$
\begin{aligned}
\frac{\partial}{\partial r}\left(\frac{1}{L_{F}} \frac{\partial L_{F}}{\partial \theta}\right) \frac{\partial}{\partial \theta}\left(\frac{1}{L_{F}} \frac{\partial L_{F}}{\partial r}\right) & \\
+ & \frac{4 a^{2} \sin ^{2}(\theta)}{\Sigma^{2}} \frac{1}{L_{F}^{2}}\left[r \frac{\partial L_{F}}{\partial r}+\cot (\theta) \frac{\partial L_{F}}{\partial \theta}\right]^{2}=0
\end{aligned}
$$

as the necessary and sufficient condition of compatibility of (28) and hence the necessary condition for the existence of solutions [27].

The field invariant $F=F_{\mu \nu} F^{\mu v}$ in the axially symmetric case reduces to

$$
F=2\left(\frac{F_{20}^{2}}{a^{2} \sin ^{2} \theta}-F_{10}^{2}\right)
$$

Equations (28) and compatibility condition (29) are satisfied by the functions [25]

$$
\begin{aligned}
& \Sigma^{2}\left(\mathscr{L}_{F} F_{01}\right)=-q\left(r^{2}-a^{2} \cos ^{2} \theta\right) ; \\
& \Sigma^{2}\left(\mathscr{L}_{F} F_{02}\right)=q a^{2} r \sin 2 \theta ; \\
& \Sigma^{2}\left(\mathscr{L}_{F} F_{31}\right)=a q \sin ^{2} \theta\left(r^{2}-a^{2} \cos ^{2} \theta\right) ; \\
& \Sigma^{2}\left(\mathscr{L}_{F} F_{23}\right)=\operatorname{aqr}\left(r^{2}+a^{2}\right) \sin 2 \theta,
\end{aligned}
$$

in two limiting cases: in the linear regime $\mathscr{L}_{F}=1$, when they coincide with the solutions to the Maxwell-Einstein equations $[39,40]$, and in the strongly nonlinear regime, when (31)-(32) satisfy system (28) as the asymptotic solutions in the limit $\mathscr{L}_{F} \rightarrow \infty$ [27]. 
In terms of the 3 -vectors defined as $[25,41]$

$$
\begin{aligned}
E_{j} & =\left\{F_{j 0}\right\} ; \\
D^{j} & =\left\{\mathscr{L}_{F} F^{0 j}\right\} ; \\
B^{j} & =\left\{{ }^{*} F^{j 0}\right\} ; \\
H_{j} & =\left\{\mathscr{L}_{F}{ }^{*} F_{0 j}\right\} ;
\end{aligned}
$$

$$
j=1,2,3
$$

the field equations (24) take the form of the source-free Maxwell equations [42]

$$
\begin{gathered}
\nabla \cdot \mathbf{D}=0 ; \\
\nabla \times \mathbf{H}=\frac{\partial \mathbf{D}}{\partial t} ; \\
\nabla \cdot \mathbf{B}=0 ; \\
\nabla \times \mathbf{E}=-\frac{\partial \mathbf{B}}{\partial t} .
\end{gathered}
$$

The electric induction $\mathbf{D}$ and the magnetic induction $\mathbf{B}$ are related to the electric and magnetic field intensities by

$$
\begin{aligned}
& D^{j}=\epsilon_{k}^{j} E^{k} ; \\
& B^{j}=\mu_{k}^{j} H^{k},
\end{aligned}
$$

where $\epsilon_{j}^{k}$ and $\mu_{j}^{k}$ are the tensors of the electric and magnetic permeability given by [25]

$$
\begin{aligned}
\epsilon_{r}^{r} & =\frac{\left(r^{2}+a^{2}\right)}{\Delta} \mathscr{L}_{F} ; \\
\epsilon_{\theta}^{\theta} & =\mathscr{L}_{F} ; \\
\mu_{r}^{r} & =\frac{\left(r^{2}+a^{2}\right)}{\Delta \mathscr{L}_{F}} ; \\
\mu_{\theta}^{\theta} & =\frac{1}{\mathscr{L}_{F}} .
\end{aligned}
$$

The relation connecting density and pressure with the electromagnetic fields reads [25]

$$
\kappa\left(p_{\perp}+\rho\right)=2 \mathscr{L}_{F}\left(F_{10}^{2}+\frac{F_{20}^{2}}{a^{2} \sin ^{2} \theta}\right) .
$$

Applying (31)-(32) in the limit $\mathscr{L}_{F} \rightarrow \infty$, we obtain

$$
\kappa\left(p_{\perp}+\rho\right)=\frac{2 q^{2}}{\mathscr{L}_{F} \Sigma^{2}} .
$$

It follows that $\mathscr{L}_{F} \rightarrow \infty$ at the $\mathcal{S}$-surface, where $p_{\perp}+\rho=$ 0 . As a result, the magnetic permeability vanishes and electric permeability goes to infinity, so that the $\mathcal{S}$-surface displays the properties of a perfect conductor and ideal diamagnetic. The magnetic induction $\mathbf{B}$ also vanishes on the $\mathcal{S}$-surface in accordance with (35), (33) and (31)-(32).

Violation of the weak energy condition within the internal cavities of the $\mathcal{S}$-surface leads to $\mathscr{L}_{F}<0$ in (37) and to negative values of the electric and magnetic permeability in (36). This can be avoided for the electrically charged regular structures satisfying the basic requirement of electrodynamics of continued media which is positivity of the electric permeability [42]. The compatibility conditions (29) admit solutions (31)-(32) for electromagnetic fields in two limiting regimes, which allows for extension of the equation state $\left(p_{\perp}+\rho\right)$ to interior cavities of $\mathcal{S}$-surface in two possible cases. The first case is similar to the shell-like models ([43] and references therein) with the flat vacuum interior, zero fields, and in consequence zero density and pressures. For the NED-GR regular structures in this case the field invariant $F$ which vanishes on the $\mathcal{S}$-surface by virtue of (31)-(32) vanishes also in its interiors, and magnetic permeability is zero at and within the $\mathcal{S}$-surface, but the electric permeability breaks from infinity on $\mathcal{S}$-surface to zero in its interior.

The second case, favored by the underlying idea of nonlinearity regularizing singularity, compatible with the existence of solutions for electromagnetic fields, and suggested by vanishing of magnetic induction on the surrounding $\mathcal{S}$ surface, is the extension of $\mathscr{L}_{F} \rightarrow \infty$ to the whole interior of the $\mathcal{S}$-surface $[26,27]$ leading to regular models with the de Sitter core, $p=-\rho$, similar to baglike models ([44] and references therein). The condition $p_{\perp}+\rho=0$ and field equations are satisfied in the limit $\mathscr{L}_{F} \rightarrow \infty$, so that the de Sitter vacuum core has the properties of a perfect conductor and ideal diamagnetic and zero magnetic induction.

4.4. Nondissipative Source of Electromagnetic Fields. On the de Sitter disk $r=0$ where $\mathscr{L}_{F} \rightarrow \infty$, we obtain from (36) $\mu_{r}^{r}=\mu_{\theta}^{\theta}=\mathscr{L}_{F}^{-1}=\mu \rightarrow 0$. The magnetic induction $\mathbf{B}$ in (35) also vanishes with taking into account (33) and (31) $[25,26]$. This suggests that an uncertainty can appear in the expression for a surface current $\mathbf{j}_{\mathbf{s}}=((1-\mu) / 4 \pi \mu)[\mathbf{n B}]$, where $\mathbf{n}$ is the normal to the surface, which could testify for transition to a superconducting state [42]. However, both these quantities, B and $\mu$, depend on $\mathscr{L}_{F}$, and to decide whether some uncertainty appears, we have to calculate the exact value of a current in the region of the maximal density.

The surface current on the disk is defined by $4 \pi j_{k}=$ $\left[e_{(k)}^{\alpha} F_{\alpha \beta} n^{\beta}\right]$ [45], where $e_{(k)}^{\alpha}$ are the base vectors related to the intrinsic coordinates on the disk $t, \phi, 0 \leq \xi \leq \pi / 2$; the vector $n_{\alpha}=\delta_{\alpha}^{1}\left(1+q^{2} / a^{2}\right)^{-1 / 2} \cos \xi$ is the unit normal to the disk, and the symbol [.]denotes a jump across its surface in the direction orthogonal to it. Using on the disk the solutions (31)-(32) and taking into account the fact that $\mu=1 / \mathscr{L}_{F}$ there, we obtain the exact expression for the surface current [26]

$$
j_{\phi}=-\frac{q}{2 \pi a} \sqrt{1+\frac{q^{2}}{a^{2}}} \sin ^{2} \xi \frac{\mu}{\cos ^{3} \xi} .
$$

Here $a$ is the radius of the disk; intrinsic coordinate on the $\operatorname{disk} \xi$ changes within $0 \leq \xi \leq \pi / 2$. The magnetic permeability 
$\mu=0$ on the disk. Therefore, the current $j_{\phi}$ is zero throughout the disk except the ring $\xi=\pi / 2$, where both terms in the second fraction go to zero independently. As a result, the surface currents on the ring can be any and amount to a nonzero total value; hence, the general condition for transition to a superconducting state [42] is satisfied.

The superconducting current (39) replaces the ring singularity of the Kerr-Newman geometry and represents a nondissipative source of the exterior fields [46], which can in principle provide a practically unlimited life time of an object [26].

4.5. Electromagnetic Soliton. The Kerr-Newman geometry inspired a flow of models of the electron since Carter found in 1968 that the parameter $a$ couples with the mass $M$ to give the angular momentum $J=M a$ and independently couples with the charge $q$ to give an asymptotic magnetic dipole moment $\mu=q a$, so that the gyromagnetic ratio $q / M$ is exactly the same as predicted for a spinning particle by the Dirac equation [39]. At the same time Carter discovered the main disaster of the Kerr-Newman geometry which is the total causality violation in the case of a charged particle when there are no Killing horizons, and any point can be connected to any other point by both a future and a past directed time-like curve [39]. Existing models involving screening or covering of causally dangerous regions require introduction of cohesive forces of nonelectromagnetic origin and are typically constructed by matching the exterior Kerr-Newman solution with some hypothetic internal sources $([47,48]$ and references therein).

Electromagnetic soliton of NED-GR describes in general setting a regular spinning object related by electromagnetic and gravitational radiation and can be applied as the model for the extended electron without additional assumption. According to Carter [39], for the electron $q=-e$ and $a=$ $\lambda_{e} / 2$, where $\lambda_{e}=\hbar / m_{e}$ is its Compton wavelength [39]. In the observer region $r \gg \lambda_{e}$, the fields are described by the Kerr-Newman limit of solutions (31)-(32) which gives [26]

$$
\begin{aligned}
& E_{r}=-\frac{e}{r^{2}}\left(1-\frac{\hbar^{2}}{m_{e}^{2} c^{2}} \frac{3 \cos ^{2} \theta}{4 r^{2}}\right) ; \\
& E_{\theta}=\frac{e \hbar^{2}}{m_{e}^{2} c^{2}} \frac{\sin 2 \theta}{4 r^{3}} ; \\
& B^{r}=-\frac{e \hbar}{m_{e} c} \frac{\cos \theta}{r^{3}} ; \\
& B_{\theta}=-\frac{e \hbar}{2 m_{e} c} \frac{\sin \theta}{r^{4}} .
\end{aligned}
$$

The Planck constant appears due to ability of the KerrNewman solution, discovered by Carter, to present the electron as seen by a distant observer. In terms of the Coleman lump, (40) describes the following situation: the leading term in $E_{r}$ gives the Coulomb law as the classical limit $\hbar=0$, and the higher terms represent the quantum corrections.

\section{Summary and Discussion}

All regular rotating objects described by the axially symmetric solutions obtained from spherical solutions with using the Newman-Janis translation are asymptotically described by the Kerr or Kerr-Newman metrics and contain de Sitter vacuum in their deepest interior (the disk $r=0$ ).

Regular rotating back holes can have at most two horizons. Geometry of the rotating black holes and solitons includes ergoregions where processes of extraction of rotational energy can occur which can explain energetic activity of a wide class of cosmic objects. For black holes, ergospheres and ergoregions exist for any density profile. In the case of a soliton, the existence of ergospheres depends on the density profile. Solitons can have two ergospheres and ergoregion between them, one ergosphere and ergoregion involving the whole interior, or no ergospheres.

Regular rotating black holes and solitons have two types of interior. The fist type of interior reduces to the de Sitter vacuum disk $r=0$. The weak energy condition is satisfied for regular objects with the interiors of the first type. The second type of interiors exists if the related spherical solution satisfies the dominant energy condition. This type of interior is presented by de Sitter vacuum $\mathcal{S}$-surface specified by $p=-\rho$, which contains de Sitter disk as the bridge. In the internal cavities of the $\delta$-surface the weak energy condition is violated.

The basic generic property of regular neutral objects described by the de Sitter-Kerr solutions is the existence of a phantom energy in the second type interiors [28].

Phantom energy, which inevitably has negative density for a certain class of observers, was introduced [32] in order to model the observed acceleration of our universe. In the most of models phantom energy originates from scalar field(s) with negative kinetic energy [49] and may involve extra dimensions (for a review [50]). For regular rotating black holes and solitons with second type interiors phantom equation of state appears without involvement of additional entities in the internal regions of strong gravitational field.

Regular rotating electrically charged black holes and spinning solitons are described by nonlinear electrodynamics coupled to gravity. For a distant observer, electromagnetic and gravitational fields asymptotically coincide with the KerrNewman fields. The nondissipative source of electromagnetic fields (which in turn form a source of gravitational fields) is a superconducting current (replacing a ring singularity) predicted in the model-independent way by dynamical equations governing regular objects related by electromagnetic and gravitational interactions.

All regular electrically charged rotating objects contain de Sitter vacuum disk $r=0$ which have the properties of the ideal conductor and ideal diamagnetic. For the objects with the second type of interiors, these properties are characteristic for the whole $\mathcal{S}$-surface. The violation of the weak energy condition in its internal cavities leads to negative values of the electric and magnetic permeability. For objects satisfying the basic requirement of electrodynamics of continued media (positivity of electric permeability), this can be avoided by extension of the equation of state $p=-\rho$ to the whole interior, 
confined by the $\mathcal{S}$-surface, in agreement with the conditions for the existence and admissible behavior of solutions for electromagnetic fields.

Mass of regular objects with the de Sitter interior is related to breaking of space-time symmetry from the de Sitter group $[18,25,33,51]$. De Sitter vacuum appears in the particle theory as a false vacuum state $p=-\rho$ of the Higgs field responsible for a particle mass. This relation allows us to explain the observable effect of the negative mass-square differences for neutrinos by applying de Sitter group for space-time symmetry in the interaction vertex and calculating particles masses as the eigenvalues of the Casimir operator in de Sitter space [52].

Experimental data reveal with a $5 \sigma$ significance the existence of a minimal length scale $l_{e}=1.57 \times 10^{-17} \mathrm{~cm}$ at the energy $E=1.253 \mathrm{TeV}$ in the annihilation reaction $e^{+} e^{-} \rightarrow \gamma \gamma(\gamma)$ [48]. It corresponds to the minimum in the $\chi^{2}$-fit and characterizes the region of the closest approach of annihilating particles specified by the finite length $l_{e}$ which cannot be made smaller. Hypotheses of quantum electrodynamics applied in the $\chi^{2}$-test assume a scattering center as a point. If the electron is an extended object, its structure would modify the QED cross-section if the test distances are smaller than its characteristic size. The results indicate decreasing cross-section with respect to that predicted by QED and testify for extended particles rather than point-like. The reaction $e^{+} e^{-} \rightarrow \gamma \gamma(\gamma)$ is purely electromagnetic and can be considered as the process of interaction of extended particles related by electromagnetic and gravitational interactions. The definite feature of the annihilation process is that at its final stage a region of interaction is neutral and spineless. It can be roughly modeled by a spherical lump with de Sitter vacuum interior $[16,18]$. For all structures with the de Sitter interior, there exists the characteristic zero gravity surface $r_{s} \simeq\left(r_{0}^{2} r_{g}\right)^{1 / 3}$ at which the strong energy condition $(\rho+$ $\sum p_{k} \geq 0$ [31]) is violated and beyond which the gravitational acceleration becomes repulsive [18]. The gravitational radius $r_{g}$ related to the energy $E=1.253 \mathrm{TeV}$ and de Sitter radius $r_{0}$ related to the Higgs vacuum expectation value responsible for the electron mass at the scale $E_{E W}=246 \mathrm{GeV}$ give $r_{*} \simeq$ $0.86 \times 10^{-16} \mathrm{~cm}$. The scale $l_{e}=1.57 \times 10^{-17} \mathrm{~cm}$ fits inside a region where gravity is repulsive and can be thus understood as a distance at which electromagnetic attraction is balanced by the gravitational repulsion of the interior de Sitter vacuum [48].

\section{Conflicts of Interest}

The authors declare that there are no conflicts of interest regarding the publication of this paper.

\section{References}

[1] S. Iso, H. Umetsu, and F. Wilczek, "Anomalies, Hawking radiations, and regularity in rotating black holes," Physical Review D: Particles, Fields, Gravitation and Cosmology, vol. 74, no. 4, Article ID 044017, 044017, 10 pages, 2006.

[2] F. Caravelli and L. Modesto, "Spinning loop black holes," Classical and Quantum Gravity, vol. 27, no. 24, Article ID 245022, 245022, 31 pages, 2010.
[3] C. Bambi and L. Modesto, "Rotating regular black holes," Physics Letters. B. Particle Physics, Nuclear Physics and Cosmology, vol. 721, no. 4-5, pp. 329-334, 2013.

[4] B. Toshmatov, B. Ahmedov, A. Abdujabbarov, and Z. Stuchlík, "Rotating regular black hole solution," Physical Review D: Particles, Fields, Gravitation and Cosmology, vol. 89, no. 10, Article ID 104017, 2014.

[5] J. C. Neves and A. Saa, "Regular rotating black holes and the weak energy condition," Physics Letters. B. Particle Physics, Nuclear Physics and Cosmology, vol. 734, pp. 44-48, 2014.

[6] S. G. Ghosh and S. D. Maharaj, "Radiating Kerr-like regular black hole," The European Physical Journal C, vol. 75, no. 1, article no. 7, 2015.

[7] S. G. Ghosh, "A nonsingular rotating black hole," The European Physical Journal C, vol. 75, no. 11, article no. 532, pp. 1-7, 2015.

[8] S. Takeuchi, "Hawking fluxes and anomalies in rotating regular black holes with a time-delay," Classical and Quantum Gravity, vol. 33, no. 22, Article ID 225016, 225016, 23 pages, 2016.

[9] T. De Lorenzo, A. Giusti, and S. Speziale, "Non-singular rotating black hole with a time delay in the center," General Relativity and Gravitation, vol. 48, no. 3, Art. 31, 22 pages, 2016.

[10] R. Torres and F. Fayos, “On regular rotating black holes," General Relativity and Gravitation, vol. 49, no. 1, Art. 2, 9 pages, 2017.

[11] E. T. Newman and A. I. Janis, "Note on the Kerr spinningparticle metric," Journal of Mathematical Physics, vol. 6, pp. 915917, 1965.

[12] M. Gürses and F. Gürsey, "Lorentz covariant treatment of the Kerr-Schild geometry," Journal of Mathematical Physics, vol. 16, no. 12, pp. 2385-2390, 1975.

[13] R. P. Kerr and A. Schild, "Some algebraically degenerate solutions of Einsteins gravitational field equations," in Proceedings of Symposium in Applied Mathematics, vol. 17, 1965.

[14] L. Modesto and P. Nicolini, "Charged rotating noncommutative black holes," Physical Review D: Particles, Fields, Gravitation and Cosmology, vol. 82, no. 10, Article ID 104035, 2010.

[15] I. Dymnikova, "Vacuum nonsingular black hole," General Relativity and Gravitation, vol. 24, no. 3, pp. 235-242, 1992.

[16] I. G. Dymnikova, "De Sitter-SCHwarzschild black hole: its particlelike core and thermodynamical properties," International Journal of Modern Physics D: Gravitation, Astrophysics, Cosmology, vol. 5, no. 5, pp. 529-540, 1996.

[17] I. G. Dymnikova, "The algebraic structure of a cosmological term in spherically symmetric solutions," Physics Letters. B. Particle Physics, Nuclear Physics and Cosmology, vol. 472, no. 1-2, pp. 33-38, 2000.

[18] I. Dymnikova, "The cosmological term as a source of mass," Classical and Quantum Gravity, vol. 19, no. 4, pp. 725-739, 2002.

[19] E. Poisson and W. Israel, "Structure of the black hole nucleus," Classical and Quantum Gravity, vol. 5, no. 12, pp. L201-L205, 1988.

[20] I. Dymnikova, "Spherically symmetric space-time with regular de Sitter center," International Journal of Modern Physics D: Gravitation, Astrophysics, Cosmology, vol. 12, no. 6, pp. 10151034, 2003.

[21] E. T. Newman, E. Couch, K. Chinnapared, A. Exton, A. Prakash, and R. Torrence, "Metric of a rotating, charged mass," Journal of Mathematical Physics, vol. 6, pp. 918-919, 1965.

[22] R. P. Kerr, "Gravitational field of a spinning mass as an example of algebraically special metrics," Physical Review Letters, vol. 11, pp. 237-238, 1963. 
[23] S. Chandrasekhar, The Mathematical Theory of Black Holes, The Clarendon Press, New York, NY, USA, 1983.

[24] A. Burinskii, E. Elizalde, S. R. Hildebrandt, and G. Magli, "Regular sources of the Kerr-Schild class for rotating and nonrotating black hole solutions," Physical Review D: Particles, Fields, Gravitation and Cosmology, vol. 65, no. 6, Article ID 064039, 064039, 15 pages, 2002.

[25] I. Dymnikova, "Spinning superconducting electrovacuum soliton," Physics Letters. B. Particle Physics, Nuclear Physics and Cosmology, vol. 639, no. 3-4, pp. 368-372, 2006.

[26] I. Dymnikova, "Electromagnetic source for the Kerr-Newman geometry," International Journal of Modern Physics D, vol. 24, no. 14, Article ID 1550094, 2015.

[27] I. Dymnikova and E. Galaktionov, "Regular rotating electrically charged black holes and solitons in non-linear electrodynamics minimally coupled to gravity," Classical and Quantum Gravity, vol. 32, no. 16, Article ID 165015, 2015.

[28] I. Dymnikova and E. Galaktionov, "Regular rotating de SitterKerr black holes and solitons," Classical and Quantum Gravity, vol. 33, no. 14, Article ID 145010, 2016.

[29] S. Coleman, "Classical lumps and their quantum descendants," in New Phenomena in Subnuclear Physics, A. Zichichi, Ed., p. 297, 1977.

[30] R. M. Wald, General Relativity, University of Chicago Press, 1984.

[31] S. W. Hawking and G. F. R. Ellis, The Large Scale Structure of Space-Time, Cambridge University Press, London, UK, 1973.

[32] R. R. Caldwell, "A phantom menace? Cosmological consequences of a dark energy component with super-negative equation of state," Physics Letters B, vol. 545, no. 1-2, pp. 23-29, 2002.

[33] I. Dymnikova, "Regular electrically charged vacuum structures with de Sitter centre in nonlinear electrodynamics coupled to general relativity," Classical and Quantum Gravity, vol. 21, no. 18, pp. 4417-4428, 2004.

[34] M. Born and L. Infeld, "Foundations of the new field theory," in Proceedings of the Royal Society of London A, vol. 144, p. 425, 1934.

[35] E. S. Fradkin and A. A. Tseytlin, "Nonlinear electrodynamics from quantized strings," Physics Letters. B. Particle Physics, Nuclear Physics and Cosmology, vol. 163, no. 1-4, pp. 123-130, 1985.

[36] A. A. Tseytlin, "Vector field effective action in the open superstring theory," Nuclear Physics. B. Theoretical, Phenomenological, and Experimental High Energy Physics. Quantum Field Theory and Statistical Systems, vol. 276, no. 2, pp. 391-428, 1986.

[37] K. A. Bronnikov, "Regular magnetic black holes and monopoles from nonlinear electrodynamics," Physical Review D: Particles, Fields, Gravitation and Cosmology, vol. 63, no. 4, Article ID 044005, 2001.

[38] I. Dymnikova, E. Galaktionov, and E. Tropp, "Existence of electrically charged structures with regular center in nonlinear electrodynamics minimally coupled to gravity," Advances in Mathematical Physics, Article ID 496475, 2015.

[39] B. Carter, "Global structure of the Kerr family of gravitational fields," Physical Review A: Atomic, Molecular and Optical Physics, vol. 174, no. 5, pp. 1559-1571, 1968.

[40] J. Tiomno, "Electromagnetic field of rotating charged bodies," Physical Review D: Particles, Fields, Gravitation and Cosmology, vol. 7, no. 4, pp. 992-997, 1973.
[41] A. Burinskii and S. R. Hildebrandt, "New type of regular black holes and particlelike solutions from nonlinear electrodynamics," Physical Review D: Particles, Fields, Gravitation and Cosmology, vol. 65, no. 10, Article ID 104017, 2002.

[42] L. D. Landau and E. M. Lifshitz, Course of Theoretical Physics, E. M. Lifshitz and L. P. Pitaevskij, Eds., vol. 9 of Statistical Physics, Part 2, Theory of the Condensed State, Pergamon Press, 1981.

[43] C. A. López, "Extended model of the electron in general relativity," Physical Review D: Particles, Fields, Gravitation and Cosmology, vol. 30, no. 3, pp. 313-316, 1984.

[44] A. Burinskii, "Gravitating lepton bag model," Journal of Experimental and Theoretical Physics, vol. 121, no. 2, pp. 194-205, 2015.

[45] W. Israel, "Source of the Kerr metric," Physical Review D: Particles, Fields, Gravitation and Cosmology, vol. 2, pp. 641-646, 1970.

[46] I. Dymnikova, "Elementary Superconductivity in Nonlinear Electrodynamics Coupled to Gravity," Journal of Gravity, vol. 2015, pp. 1-7, 2015.

[47] A. Burinskii, "Kerr-Newman electron as spinning soliton," International Journal of Modern Physics A, vol. 29, no. 26, Article ID 1450133, 2014.

[48] I. Dymnikova, A. Sakharov, and J. Ulbricht, "Appearance of a minimal length in $\mathrm{e}^{+} \mathrm{e}^{-}$annihilation," Advances in High Energy Physics, vol. 2014, Article ID 707812, 9 pages, 2014.

[49] S. M. Carroll, M. Hoffman, and M. Trodden, "Can the dark energy equation-of-state parameter $w$ be less than -1?" Physical Review D: Particles, Fields, Gravitation and Cosmology, vol. 68, Article ID 023509, 2003.

[50] K. A. Bronnikov and S. G. Rubin, "Black holes, cosmology and extra dimensions," Black Holes, Cosmology and Extra Dimensions, pp. 1-427, 2012.

[51] I. Dymnikova, "Spacetime symmetry and mass of a lepton," Journal of Physics A: Mathematical and Theoretical, vol. 41, no. 30, Article ID 304033, 2008.

[52] D. V. Ahluwalia-Khalilova and I. Dymnikova, "A theoretical case for negative mass-square for sub-eV particles," International Journal of Modern Physics D, vol. 12, no. 9, pp. 1787-1794, 2003. 


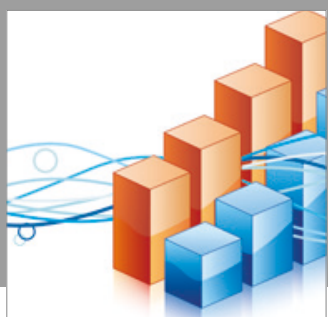

Advances in

Operations Research

vatersals

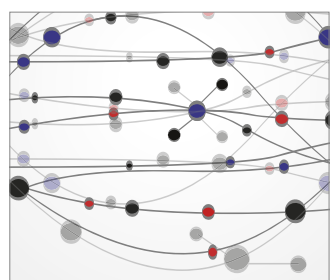

\section{The Scientific} World Journal
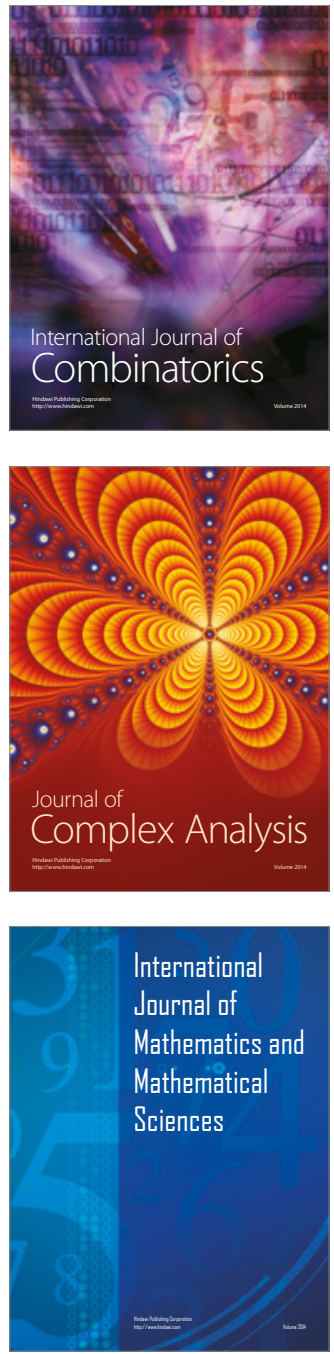
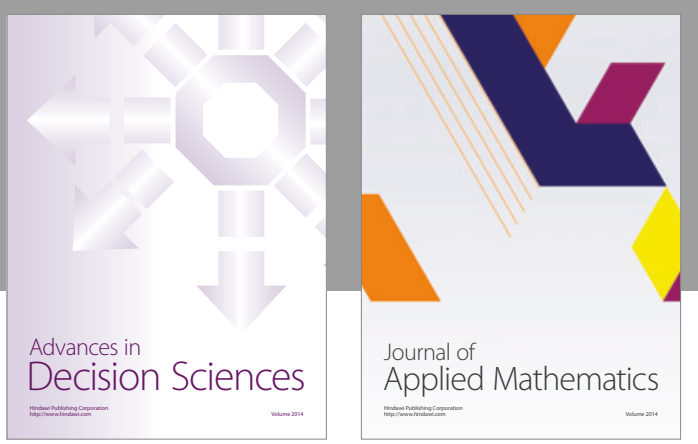

Algebra

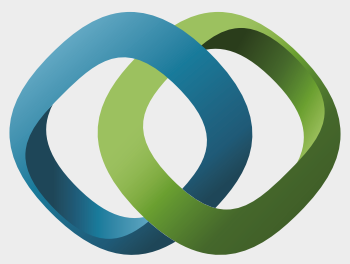

\section{Hindawi}

Submit your manuscripts at

https://www.hindawi.com
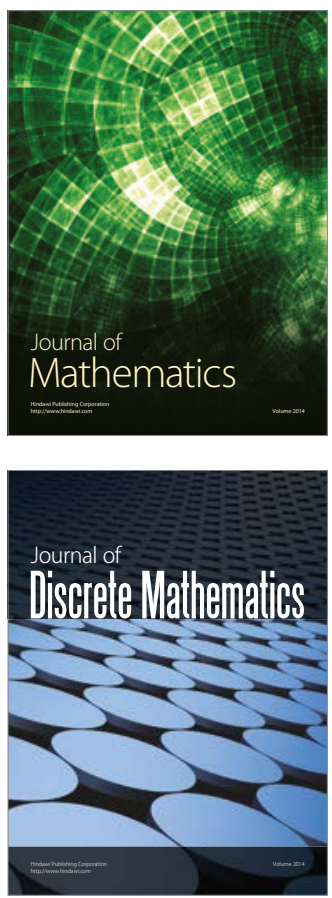

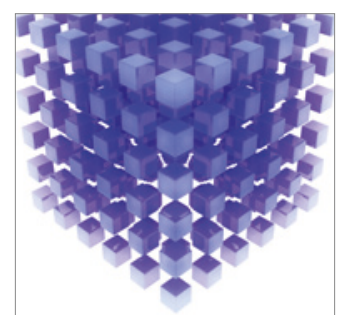

Mathematical Problems in Engineering
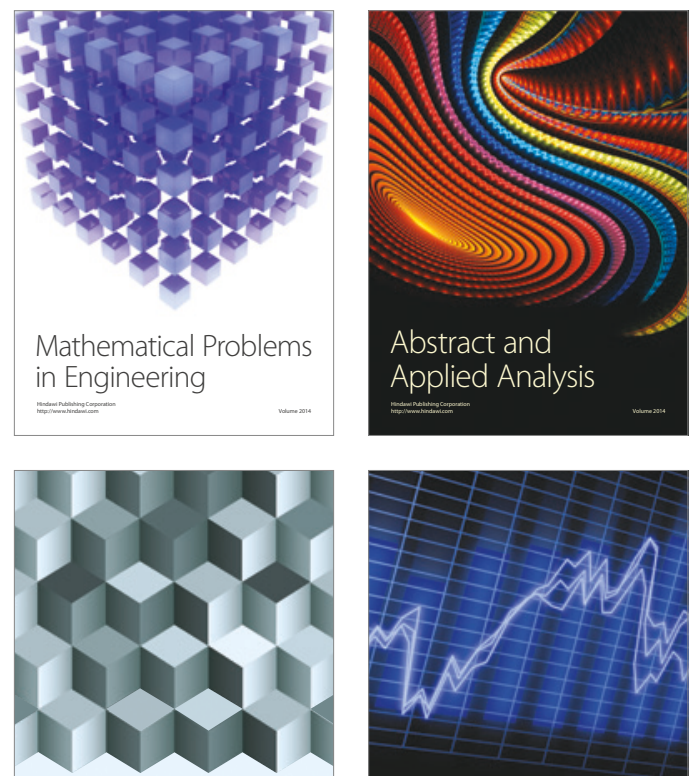

Journal of

Function Spaces

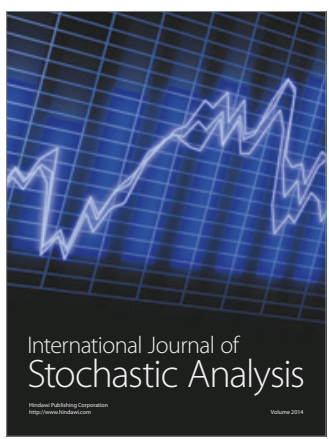

Probability and Statistics
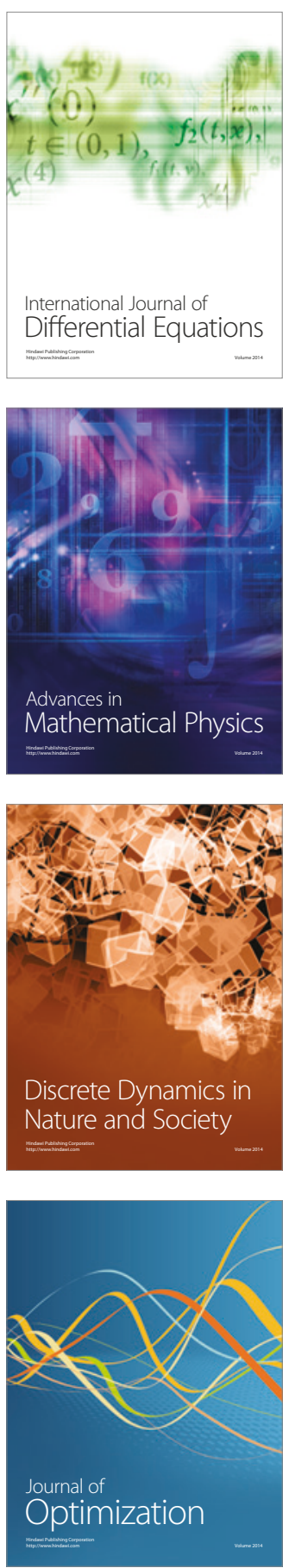